\title{
Histological lesions associated with cyclosporin: incidence and reversibility in one year old kidney transplants
}

\author{
D J VERSLUIS, F J W TEN KATE, G J WENTING, J JEEKEL, W WEIMAR
}

From the Departments of Internal Medicine and General Surgery, University Hospital, Rotterdam-Dijkzigt, and the Department of Pathology, Erasmus University, Rotterdam, The Netherlands

SUMMARY To determine the type and reversibility of the long term effects of cyclosporin A, biopsy specimens were taken from 20 recipients of kidney allografts, twelve months after transplantation, and three months later, during which time azathioprine was substituted for cyclosporin A. Arteriolar IgM and complement deposits and tubular isometric vacuolisation associated with cyclosporin A treatment significantly regressed after stopping this drug one year after transplantation. Conversion to azathioprine was accompanied by an increase in mononuclear cell infiltrates and tubulitis despite an evident improvement in renal function. Nephrotoxicity as a result of cyclosporin $A$ is common but can be reversed-at least partially.

Cyclosporin A is now widely accepted as the immunosuppressive treatment of choice in organ transplantation despite its nephrotoxic side effects. ${ }^{12}$ After kidney transplantation the adverse effect of cyclosporin A on allograft function is difficult to assess as other factors such as infection, urinary obstruction, recurrence of original nephropathy and rejection may also result in compromised renal function.

Several histopathological studies on biopsy specimens of kidney allografts with acute dysfunction have been performed in an attempt to differentiate between rejection and nephrotoxicity induced by cyclosporin $\mathrm{A}^{3-5}$ Several lesions seemed to be associated with the drug: giant mitochondria and isometric vacuolisation of proximal tubular cells, ${ }^{6-8}$ arterial hyalinosis and intimal thickening, ${ }^{67}$ mononuclear infiltrates ${ }^{3}$ and interstitial fibrosis with tubular atrophy. ${ }^{710}$ These lesions can also be found in other disorders and are not strictly diagnostic for cyclosporin A nephrotoxicity."

In a prospective trial we studied the potential reversibility of cyclosporin A nephrotoxicity in cadaveric kidney allograft recipients who had changed from cyclosporin A to azathioprine one year after transplantation. ${ }^{12}$ Renal biopsy specimens were taken before and three months after conversion, irrespective

Accepted for publication 16 December 1987 of graft function. In the present study we report on the incidence and reversibility of histopathological lesions associated with cyclosporin A after one year of continuous treatment in patients with stable graft function.

\section{Patients and methods}

Biopsy specimens were taken from 20 kidney allograft recipients. A treatment regimen of cyclosporin was started from the day of transplantation and aimed at plasma trough concentrations of $50-150 \mathrm{ng} / \mathrm{ml}$ (conventional radioimmunoassay, Sandoz Ltd, Basle, Switzerland). Patients also received prednisone (10$15 \mathrm{mg}$ ) daily. One year after transplantation all patients were admitted to our metabolic ward for evaluation of renal function. Cyclosporin A plasma trough and serum creatinine concentrations and 24 hour urinary creatinine excretion were determined. On the last day a renal biopsy was performed and cyclosporin A was stopped. The next day azathioprine was started $(2 \mathrm{mg} / \mathrm{kg} / \mathrm{day})$. At the time of conversion all patients had stable kidney function and had not experienced rejection crises in the previous six months. Three months after conversion renal evaluation was repeated including a biopsy specimen taken from those patients who had successfully converted.

Percutaneous Tru-cut needle biopsy was performed guided by ultrasound picture. The procedure was done 
only after coagulation variables were found to be adequate.

Part of the biopsy tissue was fixed in $4 \%$ formaldehyde and embedded in paraffin wax and processed for light microscopic examination. Serial 2 um thick sections were treated with haematoxylin and azafloxin, Jones, Jones Azan and CAB. Part of the biopsy specimen was snap frozen in liquid nitrogen. Cryostat sections were examined using commercial monospecific rabbit antisera directed against human IgG, IgM, IgA, IgE, C3, C1Q, fibrinogen and albumin. A control section was incubated with normal rabbit serum.

All stained sections were coded and examined by two observers who were unaware of the treatment that each patient had received. Adequacy of the biopsy specimen was judged by the number of glomeruli present. The following features were examined by light microscopy.

Glomeruli: mesangial matrix increase; basement membrane thickening; extra or intracapillary proliferation (diffuse or focal); glomerular thrombi; glomerular sclerosis; pronounced juxtaglomerular apparatus and periglomerular fibrosis.

Tubules: epithelial cell vacuolisation (isometric, anisometric) or eosinophilic degeneration; giant mitochondria; tubular casts consisting of protein, erythrocytes, or leucocytes; tubular microcalcifications; tubular basement membrane changes and tubular dilatation and atrophy.

Arterioles: endothelial cell swelling; insudative lesions (subendothelial eosinophilic fibrinoid deposits); intimal fibrosis; medial hyperplasia (symmetrical or asymmetrical).

Arteries: insudative lesions; hyalinosis; endovasculitis. Peritubular venules: dilatation.

Interstitium: infiltrates classified as diffuse or focal consisting of mononuclear, polynuclear, or eosinophilic cells, tubulitis (invasion of mononuclear cells within tubular epithelium); vasculitis; fibrosis classified as diffuse, focal, stripe-like or periglomerular; oedema; interstitial hemorrhage; calcifications.

The histopathological features were scored on a semiquantitative scale $0 /$ none, $+/$ mild, $++/$ moderate, and +++ /severe. When appropriate, only presence $(+)$ or absence $(0)$ were tabulated. Immunofluorescence microscopy not only recorded the presence or composition of immune deposits, assessed as linear or granular, but also their location at the glomerular or tubular basement membrane, mesangium, and arteriolar wall. Grading was performed according to the intensity of immunofluorescence staining on a semiquantitative scale (0 to +++$)$.

Statistical analysis was performed using the $\chi^{2}$ test contingency tables and the Student's $t$ test for paired data.

\section{Results}

CLINICAL DATA

In 16 patients elective conversion from cyclosporin $A$ to azathioprine was successfully performed, showing an improvement in renal function without signs of rejection. In two a second biopsy specimen taken at the time of re-evaluation was omitted because of a prolonged bleeding time. Consequently a renal allograft biopsy before and three months after conversion was performed in 14 patients with a stable graft function. The cyclosporin A trough concentrations before conversion were within the therapeutic range (101 (SEM 22) $\mathrm{ng} / \mathrm{ml}$ ). Serum creatinine concentration decreased in all 14 patients from 171 (13) umol/l to $133(7) \mathrm{umol} / 1$ three months after conversion $(p<0.01)$.

\section{MORPHOLOGICAL DATA}

\section{Before conversion}

Sufficient biopsy material was obtained in all 14 patients (median number of glomeruli 14, range five to 30). Glomerular changes other than mild increase in mesangial matrix and periglomerular fibrosis were uncommon before conversion one year after continuous treatment with cyclosporin A (table 1). Glomerular sclerosis $(>10 \%)$ was seen in two patients. In four biopsy specimens a prominent juxtaglomerular apparatus was found. Tubular lesions after one year of cyclosporin A treatment were predominantly located in the proximal epithelial cells (table 2). Four biopsy specimens showed isometric vacuolisation. A variable degree of anisometric vacuolisation (six patients) and eosinophilic degeneration (four patients) were also found; in three of 14 patients cytoplasmatic changes were not observed. No giant mitochondria were identified after $\mathrm{CAB}$ staining. Scattered protein casts were often seen in the tubular lumen.

In three patients red cell casts were found. Tubular microcalcifications occurred in two biopsy specimens. Thickening of basement membrane and tubular atro-

Table 1 Histopathological features of glomeruli before and three months after conversion from cyclosporin $A$ to azathioprine

\begin{tabular}{lcc}
\hline & Cyclosporin & Azathioprine \\
\hline Increase in mesangial matrix & 8 & 10 \\
Thickening of basement & 3 & 4 \\
$\quad$ membrane & 3 & 3 \\
Intracapillary proliferation & 0 & 0 \\
Extracapillary proliferation & 0 & 1 \\
Glomerular thrombi & 2 & 0 \\
Glomerular sclerosis (>10\%) & & 2 \\
Pronounced iuxtaglomerular & 4 & 2 \\
$\quad$ apparatus & 12 & 14 \\
\hline
\end{tabular}


Table 2 Histopathological features of tubuli before and three months after conversion from cyclosporin A to azathioprine

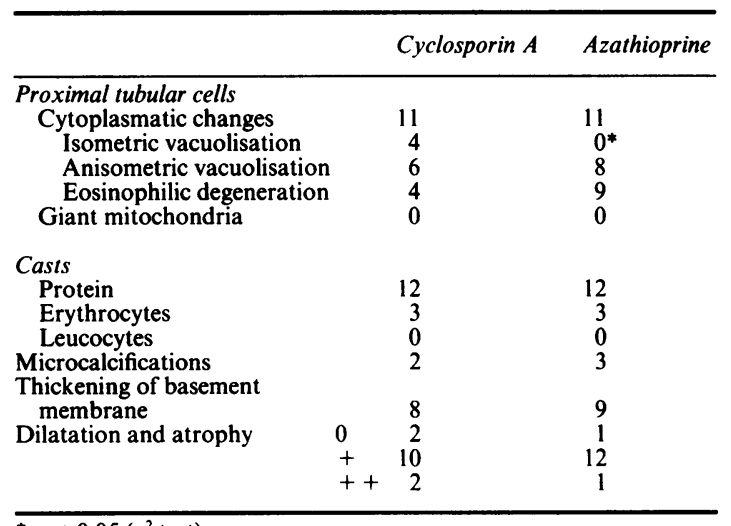

${ }^{*} \mathrm{p}<0.05\left(\chi^{2}\right.$ test $)$

phy with concomitant dilatation were present in most specimens. The degree of tubular atrophy corresponded with the extent of interstitial fibrosis ( $p<$ 0.05 ). In most patients the arterioles showed endothelial cell swelling that was associated with mild to moderate arteriolar intimal fibrosis in $50 \%$ (table 3 ). Pronounced insudative lesions were found in five patients, all of whom had a serum creatinine concentration of more than $150 \mathrm{umol} / 1(\mathrm{p}<0.05)$.

Medial hyperplasia, either symmetrical or asymmetrical, was noted in half our patients. In three a severe form of medial hyperplasia with an onion-like appearance of the vessel wall was present. No intravascular thrombi were found. The arteries showed a mild to moderate hyalinosis (table 3). Peritubular venules were dilated in four patients, but no mononuclear cells were seen inside (table 3 ).

The interstitium was the last part of the kidney examined (table 4). In 13 patients interstitial cellular infiltrates were present. They were focal and not extensive. In these infiltrates mononuclear cells predominated with a small admixture of neutrophilic and eosinophilic polymorphs. In two patients a minimal infiltration between epithelial tubular cells was seen. Interstitial fibrosis was present in all biopsy specimens, and ranged from mild to moderate. A segmental occurrence of the fibrosis caused a striped appearance in four specimens. The amount of fibrosis was associated with the extent of tubular atrophy $(\mathrm{p}<$ 0.05 ). Interstitial calcifications were present in three patients. Before conversion, circular nodular deposits of IgM and complement (C3, CIq) were prominent in the arteriolar walls of twelve patients treated with cyclosporin A (table 5). The immunofluorescence staining characteristic of glomeruli and tubuli was non-specific and weak.

\section{After conversion}

Three months after conversion from cyclosporin A to azathioprine a second biopsy was performed and the histopathological findings were compared with those by light microscopy before conversion. No changes in appearance of the glomeruli were observed (table 1). Isometric vacuolisation of the proximal tubular cells, present in four of 14 specimens before conversion, was not found after conversion ( $p<0.05$, table 2 ). No clinically important changes in anisometric vacuolisation, eosinophilic degeneration, and other tubular morphological features were observed.

In two of the five patients arteriolar insudative lesions present before conversion were still present three months after changing regimens (table 3). No changes in the incidence of endothelial cell swelling or intimal fibrosis were noted. An onion-like appearance of the arteriolar media present in three biopsy specimens before conversion was not found afterwards. The symmetrical pattern of medial hyperplasia became asymmetrical. The light microscopic appearance of the arteries and the peritubular venules was the same (table 3). A significant increase in the size of the cellular infiltrates $(p<0.01)$ characterised by an increased amount of mononuclear cells $(p<0.05)$ was present in the second biopsy specimens (table 4 ). The location of the infiltrates was still focal in 11 but had become diffuse in three patients.

An increased invasion of mononuclear cells between tubular epithelial cells showing a pronounced tubulitis occurred in nine biopsy specimens $(p<0.01)$. A local infiltration of mononuclear cells in the media and intima of arterioles was present in one patient. The pattern of interstitial fibrosis and features remained the same after conversion.

Table 3 Histopathological features of arterioles, arteries, and peritubular venules before and three months after conversion from cyclosporin A to azathioprine

\begin{tabular}{|c|c|c|}
\hline & Cyclosporin A & Azathioprine \\
\hline \multicolumn{3}{|l|}{ Arterioles: } \\
\hline Endothelial cell swelling & 10 & 12 \\
\hline Insudative lesions & 5 & 2 \\
\hline \multicolumn{3}{|l|}{$\begin{array}{l}\text { Intimal fibrosis: } \\
\text {. }\end{array}$} \\
\hline$+1++$ & 5 & 6 \\
\hline \multirow{2}{*}{\multicolumn{3}{|c|}{ Medial hyperplasia: }} \\
\hline & & \\
\hline$+1++$ & 6 & 7 \\
\hline+++ & 1 & 0 \\
\hline Onion-like & 3 & 0 \\
\hline \multicolumn{3}{|l|}{ Arteries: } \\
\hline Insudative lesions & 0 & 0 \\
\hline Hyalinosis: & & \\
\hline $\begin{array}{l}+1++ \\
+++\end{array}$ & 10 & 9 \\
\hline Endovasculitis & 0 & $\begin{array}{l}1 \\
0\end{array}$ \\
\hline \multicolumn{3}{|l|}{ Peritubular venules: } \\
\hline Dilatation & 4 & 6 \\
\hline
\end{tabular}


Table 4 Histopathological features of interstitium before and three months after conversion from cyclosporin $A$ to azathioprine

\begin{tabular}{|c|c|c|}
\hline & Cyclosporin A & Azathioprine \\
\hline \multicolumn{3}{|l|}{ Cellular infiltrates } \\
\hline \multicolumn{3}{|l|}{ Intensity: } \\
\hline $\begin{array}{l}0 \\
+\end{array}$ & $\frac{1}{6}$ & $0^{*}$ \\
\hline+ & $\begin{array}{l}0 \\
7\end{array}$ & $10^{*}$ \\
\hline$++t$ & 0 & $3^{*}$ \\
\hline \multicolumn{3}{|l|}{ Extension: } \\
\hline Focal & 13 & 11 \\
\hline Diffuse & 0 & 3 \\
\hline \multicolumn{3}{|l|}{ Composition: } \\
\hline Mononuclear cells & 13 & 14 \\
\hline Polynuclear cells & 3 & 4 \\
\hline Eosinophilic cells & 0 & 1 \\
\hline \multicolumn{3}{|l|}{ Tubulitis: } \\
\hline 0 & 12 & $5^{*}$ \\
\hline+ & 2 & $6^{*}$ \\
\hline$++1+++$ & 0 & $3^{*}$ \\
\hline Vasculitis & 0 & 1 \\
\hline \multicolumn{3}{|l|}{ Fibrosis } \\
\hline \multicolumn{3}{|l|}{ Intensity: } \\
\hline 0 & 0 & 0 \\
\hline+ & 7 & 6 \\
\hline++ & 7 & 8 \\
\hline \multicolumn{3}{|l|}{ Extension: } \\
\hline Focal & 7 & 7 \\
\hline Diffuse & 3 & 4 \\
\hline Stripe-like & 4 & 3 \\
\hline Oedema & 0 & 1 \\
\hline Interstitial hemorrhage & 0 & 0 \\
\hline Calcifications & 3 & 2 \\
\hline
\end{tabular}

${ }^{*} \mathrm{p}<0 \cdot 01$, Student's $t$ test.

Three months after conversion the arteriolar IgM and complement deposits were absent in seven and significantly less in two biopsy specimens in contrast to the 12 specimens before conversion which showed prominent immunofluorescence staining ( $p<0.05$, table 5). No association with the morphological findings of tubulitis was found. The fall in serum creatinine concentration was not related to any specific histologic feature. During a follow up period of 18 months after conversion there were no rejection episodes. Serum creatinine concentration remained stable.

\section{Discussion}

A recent editorial stated that renal biopsy specimens are far more important in ruling out rejection than determination of drug concentrations in the management of suspected cyclosporin A nephrotoxicity. ${ }^{13}$ Several histopathological studies attempted to define the diagnostic characteristics of this toxicity in renal allografts. ${ }^{3514}$ Most of these reports were of limited value because allografts with normal stable function treated with cyclosporin A were not included. When no rejection pattern was evident during periods of renal dysfunction, pronounced and unusual his- tological features were associated with cyclosporin A. ${ }^{7}$ After reducing the dose renal function improved, but biopsies were not repeated to evaluate the influence on morphological features and their potential reversibility.

In this study after one year of continuous and monitored treatment with cyclosporin A renal allograft biopsy specimens in patients with stable graft function showed no diagnostic histological markers of cyclosporin A nephrotoxicity, but several morphological features were found to be indicative of compromised renal function induced by cyclosporin $\mathrm{A}$, and their course after conversion was recorded. ${ }^{6}$

After one year of cyclosporin A treatment typical lesions included isometric vacuolisation of proximal tubular cells and arteriolopathy with an almost diagnostic IgM/C3-CIq immunofluorescence pattern. The morphological picture of tubular isometric vacuolisation is due to dilation of the smooth and rough endoplasmatic reticulum and identical with osmotic nephrosis. All tubular cells in a cross section contain densely packed empty (free of lipids) vacuoles of equal size. Isometric vacuolisation must be delineated from the vacuolisation found in ischaemic renal damage (acute tubular necrosis, vascular rejection). The latter shows vacuoles of unequal size with shedding of the cell apex. ${ }^{67}$ Proximal tubular isometric vacuolisation was found in four specimens before conversion but not after conversion. ${ }^{6}$

No giant mitochondria were identified in our series despite specific $\mathrm{CAB}$ staining. ${ }^{8}{ }^{15}$ Using lower doses of cyclosporin A, these tubular lesions are less commonly observed and were therefore probably dependent on dose. $^{67}$ The vascular changes were limited to the arterioles. The light microscopic examination showed medial cell hyperplasia and endothelial cell swelling, together with intimal fibrosis in $50 \%$ of patients. Pronounced insudative lesions were limited to patients with an increased serum creatinine concentration. Our findings agreed with a recent observation that in late biopsy specimens showed a higher incidence of

Table 5 Immunofluorescence staining characteristics of arteriolar wall before and three months after conversion from cyclosporin A to azathioprine

\begin{tabular}{lll}
\hline & Cyclosporin A & Azathioprine \\
\hline $\begin{array}{ll}\text { Arteriolar deposits } \\
\text { IgM: }\end{array}$ & \\
0 & 2 & $8^{*}$ \\
+ & 9 & $4^{*}$ \\
++ & 2 & $2^{*}$ \\
+++ & 1 & $0^{*}$ \\
Complement (C3/CIq): & 1 & 5 \\
0 & 4 & $6^{*}$ \\
+ & 5 & $3^{*}$ \\
++ & 4 & $0^{*}$ \\
+++ & 4 &
\end{tabular}


arteriolopathy in patients treated with cyclosporin A than those treated with azathioprine. ${ }^{16}$ In a recent study routine renal biopsy specimens in patients with a stable function treated with cyclosporin A showed arteriolar medial hyperplasia and arteriolar hyalinosis one month after transplantation. ${ }^{517}$ These lesions were less prominent than those in patients with renal dysfunction due to cyclosporin A nephrotoxicity the same amount of time after transplantation.

The pathogenesis of arteriolar disease associated with cyclosporin A treatment is not yet known. From studies in experimental animals and in man, it is thought that cyclosporin A may initiate or enhance vascular injury. Cyclosporin $\mathrm{A}$ accelerates arteriosclerosis in the spontaneously hypertensive rat and also enhances the vascular injury occurring in experimental acute serum sickness in rabbits. ${ }^{18} 19$ In human renal allografts the presence of glomerular capillary thrombi have been reported during treatment with cyclosporin $A .{ }^{20}$ These observations suggest that cyclosporin A causes an increase in the inflammatory component of vascular rejection, thereby leading to heavy IgM and complement deposits in the arteriolar wall. ${ }^{7}$ Three months after conversion these immunofluorescence staining characteristics were not found. We can therefore conclude that the influence of cyclosporin A on the arteriolar wall may at least be partially reversible. The results of arteriolar histological examination, however, did not differ significantly before and after conversion.

All biopsy specimens showed mild to moderate interstitial fibrosis with tubular atrophy. ${ }^{7921}$ The degree of fibrosis was strongly related to the cumulative dose of cyclosporin A during the first six months of treatment as well as to the number of acute nephrotoxic episodes. ${ }^{922}$ In our study cyclosporin A concentrations were frequently monitored and no excessive doses prescribed. In fact, aetiology and progress of fibrosis can only be adequately assessed when sequential biopsy specimens have been taken, starting at the day of transplantation..$^{23}$

A kidney already injured by other factors leading to fibrosis might be more susceptible to the effect of cyclosporin $\mathrm{A}$, but a prospective study showed no difference in the quantity of fibrosis after three months of cyclosporin A or azathioprine treatment. ${ }^{23}{ }^{24}$ When patients treated with cyclosporin A were converted three months after transplantation, a significant improvement in renal function indicated that fibrosis should not be a problem when short term administration of cyclosporin A is contemplated. During a one year follow up no increase in fibrosis leading to progressive renal insufficiency was found in the converted group. ${ }^{25}$ Our results show that even long term treatment with cyclosporin A does not lead to progressive interstitial fibrosis or vascular changes causing irreversible functional renal impairment. ${ }^{26}$

Kidney transplants with a normal function and treated with azathioprine were often found to have a prominent interstitial mononuclear cell infiltrate. ${ }^{27}$ Interstitial cell infiltrates have also been described as a prominent feature of cyclosporin A nephrotoxicity not only in renal allografts but also in normal native kidneys in patients treated with cyclosporin $A$ for uveitis. $^{3-5142728}$ After one year of cyclosporin A treatment we found a focal infiltration primarily consisting of mononuclear cells in all but one patient. ${ }^{25}$ The absence of eosinophils in these infiltrates merely excludes the possibility of a hypersensitive reaction as the cause of the interstitial nephritis. Classification of infiltrates as diffuse or local shows no relation to the degree of renal impairment. When rejection episodes occurred during treatment with cyclosporin A mostly diffuse but also focal infiltrates were reported. ${ }^{523}$ The conclusion that cellular infiltrates in renal allografts are not especially associated with cyclosporin $A$ is supported by the quantitative observation that infiltrating cells are even more numerous in grafts treated with azathioprine. ${ }^{29}$ The question of whether any infiltrate in renal allograft biopsy specimen is potentially harmful remains unanswered.

It has been suggested that cell infiltrates are all attributable to varying degrees and stages of immunological reaction to allogenic tissues. A relation between cellular rejection and so called clinical rejection is not clear cut. ${ }^{1023}$ In our study a clinically important increase in the extent of the infiltrates was found without any clinical evidence of rejection three months after conversion. The most prominent finding was an increased incidence of invasion of mononuclear cells between tubular epithelial cells.

Remarkably, infiltrating mononuclear cells invading tubular epithelium has been a hallmark of rejection for years, but now seems to be compatible with a stable graft function. ${ }^{14}$ From the excellent graft survival during our 18 months follow up it seems that inflammatory cells observed in stable allografts represent a very attenuated rejection process, perhaps only threatening the survival in the very long term. Alternatively, the mononuclear cells in functioning grafts might reflect immunological quiescence due to the development of immune regulating mechanisms involving circulating antibodies and suppressor cells. The invasion of mononuclear cells between tubular epithelium prove that these cells are not quiescent but really represent an active cell population. Development of a florid clinical rejection episode shortly after conversion suggests that in some patients there is a rapid proliferation of cytotoxic effector cells on withdrawal of cyclosporin A, which escapes the regulating mechanisms. ${ }^{12}$ The clinical improvement in renal function after conversion seems to be due to abolition of 
cyclosporin A nephrotoxicity rather than control of a low grade rejection process.

In conclusion, our study showed that histopathological features indicative of compromised renal function induced by cyclosporin $\mathbf{A}$ include isometric proximal tubular cell vacuolisation and arteriolar lesions. This arteriolar disease consists of insudative lesions and IgM/complement deposits. After changing to azathioprine no isometric vacuolisation was found and the arteriolar deposits of IgM/complement were considerably less. Mild to moderate degrees of interstitial fibrosis do not interfere, at least in part, with the reversibility of graft dysfunction, but conversion is accompanied by an increase in mononuclear cell infiltration, together with tubulitis. These remarkable morphological findings, combined with the clinical improvement of renal function in all patients observed, is a new contribution to the discussion of the hazards of long term treatment with cyclosporin A.

We thank Mrs W Zuidema for secretarial assistance.

\section{References}

1 European Multicentre Trial Group. Cyclosporin in cadaveric renal transplantation: one-year follow-up of a multicentre trial. Lancet 1983;ii:986-9.

2 Bennett WM, Pullian JP. Cyclosporine nephrotoxicity. Ann Intern Med 1983;99:851-4.

3 Sibley RK, Rynasiewicz J, Ferguson RM, et al. Morphology of cyclosporin nephrotoxicity and acute rejection in patients with immunosuppressed with cyclosporin and prednisone. Surgery 1983;94:225-34.

4 Klintmalm G, Bergstrand A, Ringden O, et al. Graft biopsy for the differentiation between nephrotoxicity and rejection in Cyclosporin A-treated renal transplant recipients. Transplant Proc 1983;15:493-6.

5 Neild GH, Taube DH, Hartley RB, et al. Morphological differentiation between rejection and cyclosporin nephrotoxicity in renal allografts. J Clin Pathol 1986;39:152-9.

6 Bergstgrand A, Bohman O, Farnsworth A, et al. Renal histopathology in kidney transplant recipients immunosuppressed with cyclosporin A: results of an international workshop. Clin Nephrol 1985;24:107-19.

7 Mihatsch MJ, Thiel G, Spichtin HP, et al. Morphological findings in kidney transplants after treatment with cyclosporin. Transplant Proc 1983;15(suppl 1):2821-35.

8 Kirwan PD, Baxter CR, Duggin GG, et al. Giant mitochondria, renal transplant biopsy, and cyclosporin A. Lancet 1981;ii: 146-7.

9 Klintmalm G, Bohman SO, Sundelin B, Wilczek H. Interstitial fibrosis in renal allografts after 12 to 46 months of cyclosporin treatment: beneficial effect of low doses in early post-transplantation period. Lancet 1984;ii:950-4.

10 Farnsworth A, Hall BM, Duggin GG, et al. Renal biopsy morphology in renal transplantation. A comparative study of the light microscopic appearances of biopsies from patients treated with cyclosporin A or azathioprine, prednisone and antilymphocyte globulin. Am J Surg Pathol 1984;8:243-52.
11 Wallace AC. Histopathology of cyclosporin A. Transplant Proc 1985;17(suppl 1):117-22.

12 Versluis DJ, Wenting GJ, Derkx FHM, et al. Who should be converted from cyclosporin to conventional immunosuppression in kidney transplantation, and why. Transplantation 1987;44:387-9.

13 Strom TB, Loertscher R. Cyclosporine induced nephrotoxicity. $N$ Engl J Med 1984;311:728-9.

14 Verani RR, Flecher SM, Van Buren CT, et al. Acute cellular rejection or cyclosporin A nephrotoxicity? A review of transplant renal biopsies. Am J Kidney Dis 1984;IV:185-91.

15 Mihatsch MJ, Olivieri V, Marber U, et al. Giant mitochondria in renal tubular cells and cyclosporin A. Lancet 1981;i:1162-3.

16 Gokel JM, Stoffner D, Castro LA, Hillebrand G, Land W. Late biopsy and clinical findings in renal transplant recipients under cyclosporin treatment (comparison with azathioprine-treated patients). Transplant Proc 1986;18:1005-8.

17 Taube DH, Neild GH, Gwyn Williams D, et al. Differentiation between allograft rejection and cyclosporin nephrotoxicity in renal-transplant recipients. Lancet 1985;ii:171-4.

18 Ryffel B, Siegl H, Mueller AM, Hauser R, Mihatsch MJ. Nephrotoxicity of Cyclosporin in spontaneously hypertensive rats. Transplant Proc 1985;18:1430-1.

19 Neild GH, Ivory K, Williams DG. Glomerular thrombi and infarction in rabbits with serum sickness following cyclosporin therapy. Transplant Proc 1983;15(suppl 1):2782-6.

20 Neild GH, Reuben R, Hartley RB, Cameron JS. Glomerular thrombi in renal allografts associated with cyclosporin treatment. J Clin Pathol 1985;38:253-8.

21 Hamilton DV, Calne RY, Evans DB, Henderson RG, Thirn S, White DJG. The effect of long-term cyclosporin on renal function. Lancet 1981;i:1218-9.

22 Bohman SO, Klintmalm G, Ringden O, Sundelin B, Wilczek H. Interstitial fibrosis in human kidney grafts after 12 to 46 months of cyclosporin therapy. Transplant Proc 1985;17:1168-71.

23 d'Ardenne AJ, Dunnill MS, Thompson JF, McWhinnie D, Wood RFM, Morris PJ. Cyclosporin and renal graft histology. J Clin Pathol 1986;39:145-51.

24 Wood RFM, Thompson JF, Allen NH, Ting A, Morris PJ. The consequences of conversion from cyclosporin to azathioprine and prednisolone in renal allograft recipients. Transplant Proc 1983;15(suppl 1):2862-8.

25 d'Ardenne AJ, Dunnill MS, Wood RFM, Thompson JF, Morris PJ. Cyclosporin treatment does not cause specific histologic changes in human renal allograft. Transplant Proc 1985; 17:1166-7.

26 Schmidt U, Mihatsch MJ, Albert FW. Morphologic findings in kidney transplants one year after treatment with cyclosporin A (CyA) alone or in combination with low-dose steroids. Transplant Proc 1986;18:1266-8.

27 Burdick JF, Beschorner WE, Smith WJ, et al. Characteristics of early routine renal allograft biopsies. Transplantation 1984; 38:679-84.

28 Palestine AG, Austin HA, Balow JE, et al. Renal histopathological alterations in patients treated with cyclosporin for uveitis. $N$ Engl J Med 1986;314:1293-8.

29 McWhinnie DL, Thompson JF, Taylor HM, et al. Leukocyte infiltration patterns in renal allografts assessed by immunoperoxidase staining of 245 sequential biopsies. Transplant Proc 1985;17:560-1.

Requests for reprints to: D J Versluis, University Hospital Rotterdam-Dijkzigt, Room D 408, Dr. Molewaterplein 40, 3015 GD Rotterdam, The Netherlands. 